\title{
EDITORIAL
}

\section{'Allogeneic marrow transplantation in children with acute leukemia: a practice whose time has gone': twenty years later}

Leukemia (2009) 23, 2189-2196; doi:10.1038/leu.2009.132

In June 1988, I discussed some critical aspects of Leukemia therapy at the Modern Trends in Leukemia symposium in Wilsede, Germany. Included were comments about myeloablation and allogeneic hematopoietic cell transplantation (alloHCT) following chemotherapy (chemo) in children with acute leukemia. ${ }^{1}$ While speaking restlessness was apparent among clinicians in the audience. Immediately after I concluded the chairman quickly announced there would be no questions and adjourned the meeting for dinner.

As I departed the building among murmurs, I met Dr Nicole Muller-Berat Killmann, Editor of the journal Leukemia. She asked me to submit my comments about allo-HCT to Leukemia with the understanding that respected colleagues using alloBMT would be invited to respond. The commentary appeared several months later followed by thoughtful responses from Drs Judith Chessells and John Kersey. ${ }^{2,3}$

The commentary and subsequent updates in 1990 and 1993 summarized published reports about the relative efficacy of chemo alone or chemo followed by allo-HCT in children with acute leukemia in remission. ${ }^{4,5}$ It was noted that most reports showed no advantage of adding allo-HCT to chemo because treatment-related mortality cancelled out any advantage in fewer relapses. Reports of its superiority over combination chemo probably resulted from selective bias in choosing patients for allo-HCT, such as delay between remission and transplant. Publication bias was also a possible source of error. ${ }^{5}$ In addition, allo-HCT failed to achieve a primary goal of curative therapy of children: restoration of normal health with normal capacity for growth and development. Children surviving the immediate mortality of allo-HCT experienced numerous and continuing sequelae, including somatic growth failure, chronic graft versus host disease (GVHD), multiple endocrine disorders, second cancers, sterility, renal insufficiency, obstructive and restrictive pulmonary disease, aseptic necrosis of bone and leukoencephalopathy. ${ }^{6,7}$ The medications used for GVHD also carried additional toxicities. Decisions about whether to subject children to allo-HCT needed to weigh in these risks versus those of a specific chemo program alone. The commentary concluded that Allo-HCT did not qualify for treatment of acute leukemia in children, only as a research procedure to be conducted under the same regulations as any other. Furthermore, the number and complexity of risk factors for treatment failure in children with acute leukemia and the variety of previous treatments required a randomized comparison of benefit and risk in a similar cohort of children, in remission after standardized chemo, who have HLA-matched donors. Randomization to continued chemo or allo-HCT would be made immediately before the scheduled date of a standardized allo-HCT procedure to avoid time to transplant and transplant method bias.

Such a randomized study has never been reported. Comparison of chemo and chemo plus allo-HCT has used time from diagnosis or remission to transplant, HLA-matched donor availability and intent-to-treat, historical patient risk factors for treatment failure and matched pairing of transplanted and nontransplanted patients in attempts to accommodate the lack of randomization. However, reported comparisons are often impeded by variable chemo schedules and allo-HCT methods. Many risks for treatment failure are unaccounted for such as socio-economic and insurance status, ethnicity, geographic residence and venue of patient care. Children receiving alloHCT are usually treated in larger well-staffed centers with more abundant resources. Those receiving continued chemo are more often cared for in small less-gifted facilities. In addition, most reports fail to provide detailed information about late sequelae such as growth and organ failure and second cancers. For this reason conclusions cannot be drawn about risk/benefit ratios of chemo versus chemo and allo-HCT, an essential need in determining the relative value of alternate treatment methods, especially in children with expectation of long lives and the challenges of growing to happy and healthy adults.

Despite the lack of proof that allo-HCT increases the true cure rate of children with acute leukemia and the reports of its serious immediate and late morbidity and mortality it has become a widespread practice, especially in economically privileged nations. The purpose of this review and commentary is to revisit the thesis proposed 20 years ago: allogeneic marrow transplantation in children with acute leukemia is a practice whose time has gone.

The acronym allo-HCT is used here for matched-related donor, usually sibling, hematopoietic transplantation. Other methods of HCT are often employed that use matched unrelated donors, haploidentical donors, umbilical cord stem cells and stored purged autologous bone marrow. Because related donor allo-HCT is generally considered the standard this review will discuss, as possible, only comparisons of this modality with continued chemotherapy. To attempt some clarity the comparisons are reviewed and discussed with regards to three situations: acute lymphoblastic leukemia (ALL) historically at high risk of treatment failure in first remission, ALL in second remission after failure of initial treatment and acute myeloid leukemia (AML) in first remission.

Survival has improved in these children with both chemo and chemo plus allo-HCT over the years with more experience and better resources and staffing of pediatric hematology/oncology services, so only reports of the last decade are reviewed here. For a thorough detailed review of HCT in children with ALL please consult the 2005 report commissioned by the American Society of Blood and Bone Marrow Transplantation. ${ }^{8}$

\section{ALL in first remission at high risk of treatment failure}

Infants less than 1 year old with ALL that shows an $11 \mathrm{q}$ chromosome translocation and rearrangement of the myeloid/ lymphoid or mixed lineage leukemia gene have a poor outlook for survival and cure. An international collaborative retrospective study by Pui et al. ${ }^{9}$ of 256 infants with $t(4 ; 11)$ ALL used 
statistical adjustments for initial leukocyte count, age and waiting time to transplant to compare chemo alone and HCT. It showed that the 68 patients, 56 of whom had t(4:11) ALL, who received HCT of any kind, including allo-HCT, had less diseasefree and overall 5-year survival than those who received chemo only (Figure $1 \mathrm{a}$ and b). Supporting this finding, Chessells et al. ${ }^{10}$ reported the results of a UK infant ALL study in the same year. Adjusting for delay to transplant, 5-year event-free survival was similar for 12 patients with ALL showing 11 q23 abnormality who received matched sibling or unrelated allo-HCT and 57 who received continued chemo. As with other infant studies total body irradiation was omitted in the myeloablation

a

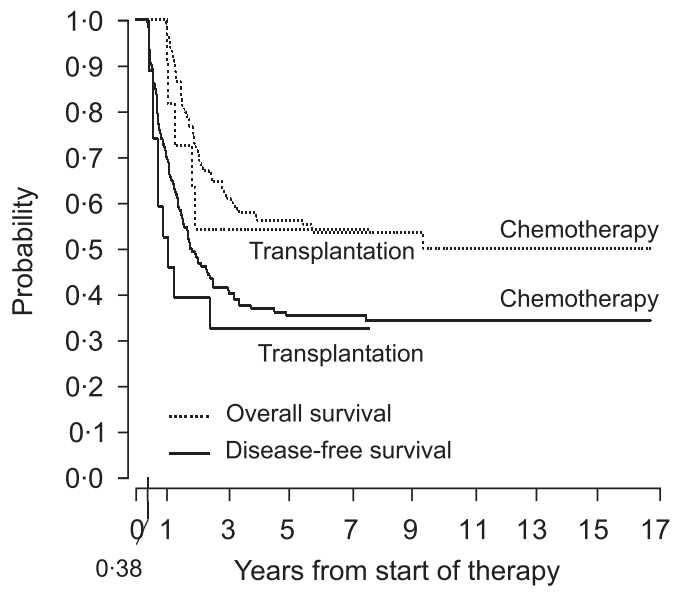

Patients at risk

Overall survival

Chemotherapy

Transplantation

Disease-free survival

Chemotherapy

Transplantation

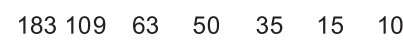

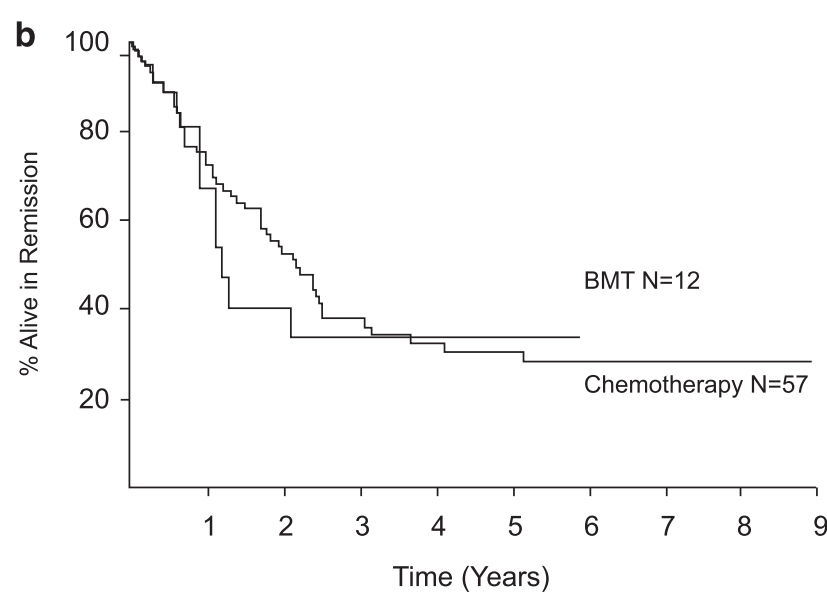

Figure 1 (a) Estimated disease-free survival and overall survival of infants with $\mathrm{t}(4 ; 11) \mathrm{ALL}$ in first remission treated with chemo alone or chemo and allo-HCT are similar. Delay from remission to transplant was accounted for by Mantel-Byar estimate of disease-free survival (DFS) with a landmark of 0.38 year and in overall survival (OAS) by Kaplan-Meier estimate with a landmark of 1 year. (From Pui et al., The Lancet 2002; 359:1913. Reproduced with permission). (b) Comparison of estimated EFS of infants with ALL in remission with $11 \mathrm{q} 23$ abnormality who received allo-HCT or continued chemo from median time to transplant. There was no significant difference whether or not the chemo group included only infants with 11q23 ALL (From Chessells et al., British Journal of Hematology 2002; 117: 306. Reproduced with permission). procedure because of its effect on growth. This might reduce the efficacy of the allo-HCT procedure.

In a 2007 report Pieters et al. ${ }^{11}$ confirmed these observations. They described an international multicenter trial of therapy of 402 infants with ALL younger than 1 year of age. A total of 41 infants at high risk of treatment failure received HCT following remission induction by intensive chemo. When adjusted for waiting time to HCT their 4-year disease-free survival did not differ significantly from that of comparable patients treated with continued chemo.

In 2005 Balduzzi et al. ${ }^{12}$ reported the results of a planned multi-institutional comparison of allo-HCT versus continued chemo in children with ALL at a very high risk of treatment failure as defined by initial remission induction failure, poor response to prednisone, $t(9 ; 22)$ or $t(4 ; 11)$ clonal abnormalities or initial leukocyte count $>100000 /$ ul. Children were allocated to treatment with continued chemo or allo-HCT in accordance with availability of a compatible related donor and the outcome was analyzed by 'intent to treat'. For 280 children assigned to continued chemo the 5 -year disease-free survival was $40.6 \%$ and overall survival $50.1 \%$. For 77 children who received alloHCT the 5-year disease-free survival was $56.7 \%$ and overall survival $56.4 \%$. Although the event-free survival was statistically significantly better for allo-HCT patients, the overall survival was not, possibly due to better response to 'salvage' chemo for the chemo patients. This emphasizes the importance of reporting overall survival as well as event-free and disease-free survival. Unfortunately, this is neglected in many publications comparing chemo and allo-HCT in children with leukemia.

About $3-5 \%$ of childhood ALL display a 9;22 chromosomal translocation (Philadelphia chromosome or $\mathrm{Ph}+$ ) and contain the associated hybrid BCR-ABL gene and protein. Mortality has been high on traditional ALL therapy, so that allo-HCT has been widely used after initial chemo-induced remission. A retrospective international survey of children and young adults by Arico et al. ${ }^{13}$ compared the outcome in 147 patients who received chemo alone with 38 who received allo-HCT after chemo-induced remission. Five-year disease-free and overall survival was significantly superior with allo-HCT in all three prognosis groups as classified by age and initial leukocyte count. However, a more recent retrospective survey by the same investigators of 640 similar patients with $\mathrm{Ph}+\mathrm{ALL}$ treated between 1995 and 2005 yielded a different result. ${ }^{14}$ The 7 -year overall survival rate of 65 patients in remission who received chemo followed by allo-HCT was not significantly different from that of the patients who received continuing chemo. The authors suggest, like Balduzzi et al. ${ }^{12}$ that successful 'salvage' chemo after relapse in the chemo alone group was accountable.

In a recent Children's Oncology Group prospective study, the tyrosine kinase binder imatinib mesylate was included in the chemo schedule for children and youth with $\mathrm{Ph}+$ ALL. ${ }^{15}$ The 3-year event-free survival was compared by 'intention to treat' for 21 patients who received allo-HCT while in remission and 25 patients treated with 40 weeks of continued chemo including 40 weeks of imatinib. Survival was not significantly different between the two groups. Historical comparison of the imatinib-containing chemo with past experience of the group before its use suggested that its inclusion doubled the event-free survival of children and youth with $\mathrm{Ph}+\mathrm{ALL}$. The addition of imatinib did not seem to increase risks of drug toxicity. The authors conclude that matched sibling donor allo-HCT does not appear to offer a benefit to early survival of children and youth with $\mathrm{Ph}+\mathrm{ALL}$ as compared with continued chemo that includes 40 weeks of imatinib. The report also suggests that patients with $\mathrm{Ph}$ negative 
ALL with poor response to conventional induction therapy had better early outcomes when imatinib was included in their chemo.

About $1 \%$ of children with ALL have hypodiploid leukemia cells with less than 45 chromosomes and a lower cure rate. Nachman et al. ${ }^{16}$ reported that nine patients with ALL showing this feature received HCT during first remission. Event-free and overall survival did not differ significantly between them and other ALL patients with the same feature who did not receive $\mathrm{HCT}$.

In conclusion, the results of infant ALL studies are consistent in demonstrating that allo-HCT has no place in their treatment, although the necessary omission of total body irradiation in myeloablation may compromise its effectiveness. With regard to $\mathrm{Ph}+\mathrm{ALL}$ there has been little evidence that allo-HCT has much influence on long-term survival compared with chemo alone. The treatment-related mortality of allo-HCT cancels out any advantage in less relapse. With the addition of imatinib to the chemotherapy it now appears that there is no reason to employ allo-HCT in initial treatment of children with $\mathrm{Ph}+\mathrm{ALL}$. With regard to other types of ALL at high risk of treatment failure the reports by Balduzzi et al. ${ }^{12}$ and Nachman et al. ${ }^{16}$ suggest lack of overall survival advantage of allo-HCT in a variety of children with historically poor prognosis for cure.

\section{ALL in second remission after relapse following first remission}

In the past, children with ALL in relapse often had lasting remissions and cure when they received modern curative treatment after having had a relapse on palliative chemotherapy. Today, those who experience relapse, particularly initial hematological relapse, after modern combination chemotherapy have much less opportunity for survival and cure because of drug-resistant disease. ${ }^{17}$ Allo-HCT has been widely and increasingly used in these children to supplement 'salvage' chemo for almost 40 years because it offers a graft versus leukemia effect as well as myeloablative chemo and, most often, total body irradiation, another antileukemic agent.

Following are summaries of reports published during the past decade that compare allo-HCT with continued chemo in children who are in second remissions of ALL after relapse following initial treatment.

In a UK study by Lawson et al. ${ }^{18}$ the relative benefit of alloHCT and continued chemo was determined in a cohort of 246 children with ALL in second remission by matched donor availability comparison. For children allocated to allo-HCT the 7 -year event-free survival was $45 \%$ and for those assigned to continued chemo it was $39.7 \%$, not a significant difference when adjusted for time to transplant, duration of first remission, marrow involvement at relapse and age at diagnosis (Figure 2). Late morbidity and mortality were not compared. The authors suggest reconsideration of the practice of using allo-HCT for children with relapsed ALL.

In a 2006 retrospective analysis by Eapen et al. ${ }^{19}$ of children with B-precursor ALL in second remission, the IBMTR center compared outcomes of patients enrolled in Children's Oncology Group studies who received allo-HCT or continued chemotherapy. Outcomes were corrected for time to transplant bias. For 186 children who received allo-HCT and 188 continued chemo 8-year leukemia-free survival was significantly greater with allo-HCT for those with early relapse who received total body irradiation (TBI) in the myeloablation phase. However, for those with late relapse event-free survival was not significantly different. For patients whose allo-HCT procedure excluded TBI

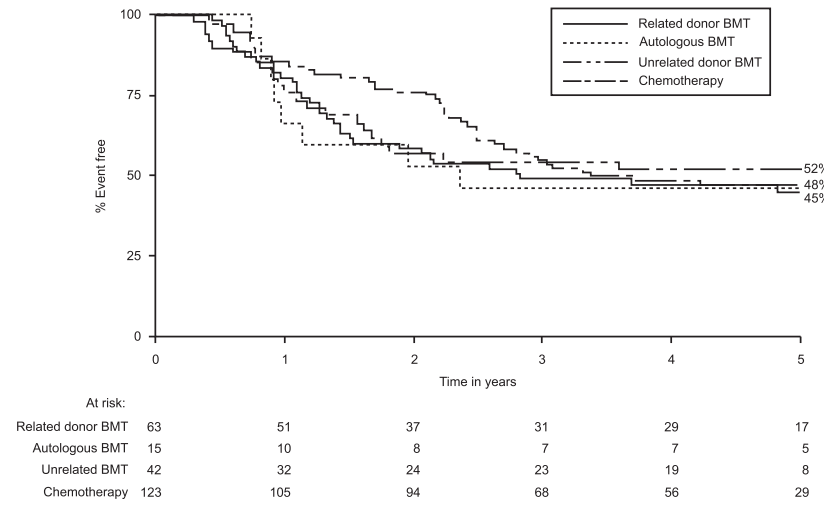

Figure 2 Estimated EFS from first relapse by treatment received in second remission of children with ALL. Adjusted for time to transplant, duration of first remission and site of relapse, EFS was not significantly different for the children whether treated with continued chemo or with HCT from related or unrelated donors or with autologous HCT (From Lawson et al., British Journal of Hematology 2000; 108: 531. Reproduced with permission).

survival was inferior. Overall survival and late sequelae are not described. The report concludes that allo-HCT including TBI offers a 'clear advantage' over continued chemo in children with early relapse of B-precursor ALL in second remission. However, the lack of information about overall survival raises the question whether this advantage was reflected in cure rate.

The BFM group led by Einsiedel et al. ${ }^{20}$ conducted a prospective study to determine a uniform treatment for children with relapsed ALL. The 188 children who experienced a second remission were stratified into early relapse, late relapse and isolated extramedullary relapse groups. Allo-HCT including TBI was used for those with matched donors. Twenty-seven children with early or late relapse received allo-HCT. Their 15-year event-free survival, adjusted for delay of transplant, was significantly better than those who received continued chemo combined with preventive cranial irradiation. Comparison of overall survival and eventual plateau of survival was not reported. The authors point out the need to clarify indications for allo-HCT and that its long-term sequelae as well as its acute mortality and toxicity need to be taken into account. They stop short of recommending it.

A Nordic report by Schroeder et al. ${ }^{21}$ compared the survival of 75 children with ALL in second remission who received alloHCT between 1981 and 1995 with those who received only continued chemo. Two chemo-treated patients with similar risks for second relapse were matched with each allo-HCT-treated patient. Eight-year event-free survival was significantly greater for allo-HCT than chemo patients among those who had relapsed while receiving initial therapy or within 6 months of its cessation. However, for those who relapsed later the outcome was not significantly different. Again, overall survival estimates are not provided. A more recent population-based Nordic report of children with ALL demonstrates that the pathway leading to allo-HCT in second remission is 'a remarkable selection process' ${ }^{22}$ The report emphasizes the need to understand this in evaluating allo-HCT versus chemo.

Three reports from cooperative childhood cancer groups published in the past 4 years provide evaluations of the survival benefit of allo-HCT in children with ALL in second remission treated with more contemporary resources and staffing.

A UK group made a retrospective analysis of 139 children in second remission of ALL after relapse on the same initial treatment. ${ }^{23}$ Patients were stratified by an estimated risk for a 
second relapse; 32 received allo-HCT. For high-risk patients allo-HCT appeared to benefit survival although time to transplant was not considered. For intermediate risk patients there appeared to be no advantage. None of the standard risk patients received allo-HCT. Estimated event-free and overall survival rates for patients who received allo-HCT are not reported.

A Children's Oncology Group (COG) study by Malenpati et al. ${ }^{24}$ described 347 children with 'standard risk' ALL as defined by initial leukocyte counts and age who experienced relapse after initial treatment on the same regimen. Of 89 patients treated in second remission with HCT, the hematopoietic cell source was certain in 50 patients. Twenty received matched sibling cells, 14 matched unrelated and 14 unrelated umbilical cord cells. After adjusting for therapy selection bias the event-free survival and overall survival of children in complete remission who received allo-HCT and that of the children who had continued chemo were similar (Figure 3).

Gaynon et al..$^{25}$ reported a Children's Oncology Group prospective study of 155 children with ALL in second remission after hematological relapse within 12 months of cessation of initial chemo. Using intent-to-treat analysis they compared 3-year and 5-year estimated disease-free survival from remission induction of 50 patients with matched-related donors, 37 with matched unrelated, haploidentical related or autologous donors and 35 who received chemo alone. There was no significant difference in the survivals of the three groups of patients (Figure 4). The authors point out that comparisons of this type reflect specific patients and specific treatments and may not extrapolate to general conclusions. This re-emphasizes the need for truly randomized comparisons of patients with similar host and disease features who receive similar chemotherapy and standardized allo-HCT.

To summarize, the benefit of adding allo-HCT to the chemo of ALL in second remission is questionable whereas the long-term and continuing risks of myeloablation, commonly including TBI, such as growth and organ failure and GVHD are considerable in the growing child. Lack of follow-up information about the comparative risks of allo-HCT and continued chemo in treatment of childhood ALL prevents assessment of risk/benefit ratios and hinders decisions about its relative advantage over chemo alone in pediatric practice.

\section{Acute myeloid leukemia in first remission}

When curative chemotherapy for childhood acute leukemia was introduced 40 years ago it soon became apparent that it was largely ineffective in curing children whose leukemias originate from early myeloblasts, such as acute myeloblastic, monoblastic and myelomonoblastic leukemia. Here, we will use the eponym AML for acute leukemias of myeloid cell origin. Because of the failure to cure AML with chemo, allo-HCT was turned to for treating these diseases and became popular in the 1980s. ${ }^{5}$ However, simultaneously, drugs were developed that were more efficacious against AML so that by 1993 several nonrandom comparisons of $\mathrm{AML}$ treated with continuing chemo and chemo plus allo-HCT showed equivalent leukemia survival when corrected for selection factors.

As with ALL experience, cure rates of AML have gradually improved since then, mostly because of advances in supportive therapy, better resources and more and better trained and experienced pediatric hematology staff. Cure rates for childhood AML have improved both with chemo and chemo plus allo-HCT. Several reports of cooperative studies that compare
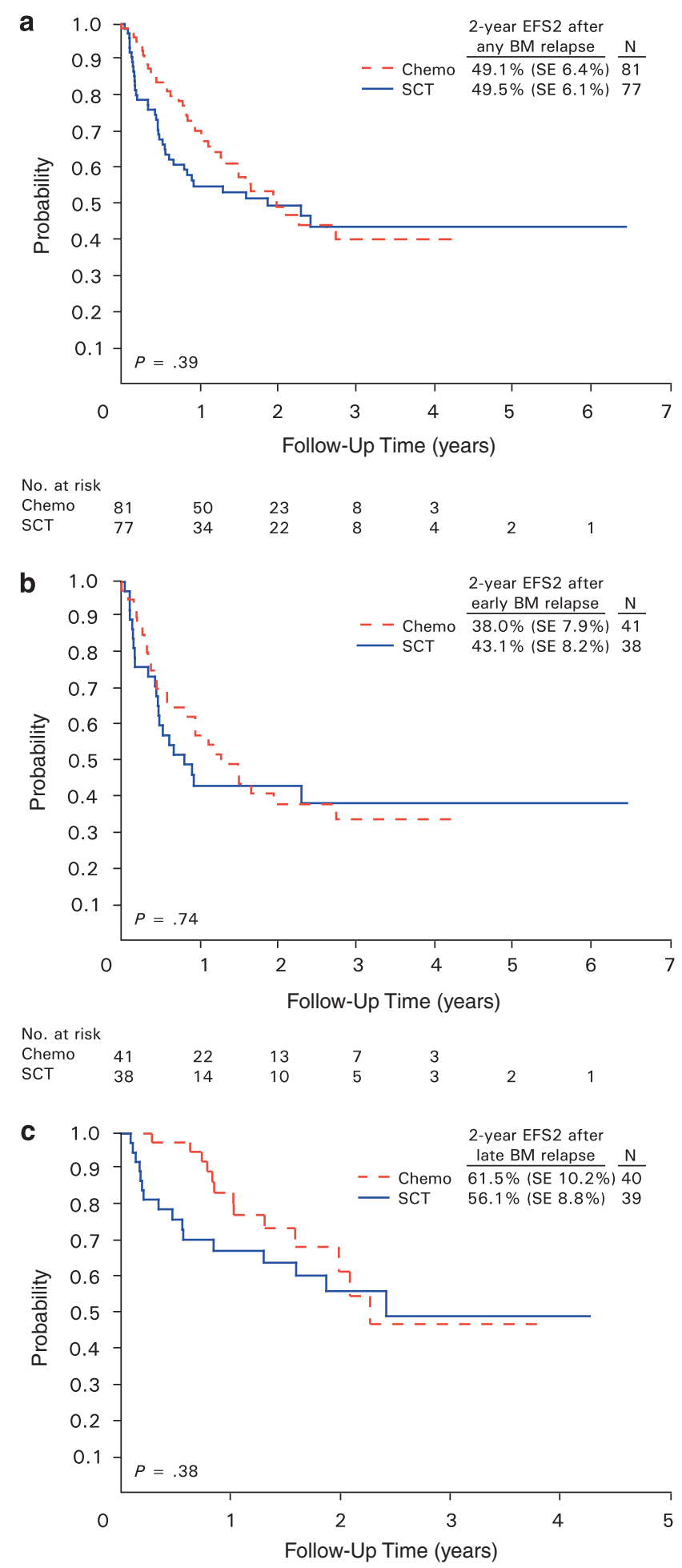

No. at risk

Chemo 40

SCT $\quad 39$ 28 20

9
12

1

Figure 3 Estimated EFS of children with historically 'standard risk' ALL in second remission who received HCT or chemo alone. The HCTs were predominantly from matched siblings but some were from matched unrelated donors and some consisted of unrelated umbilical cord blood. Kaplan-Meier estimates of 2-year EFS adjusted for time to transplant were similar for (a) any bone marrow relapse, (b) early bone marrow relapse and (c) late bone marrow relapse (From Malapenti et al., Journal of Clinical Oncology 2007; 25: 5800. Reproduced with permission). 


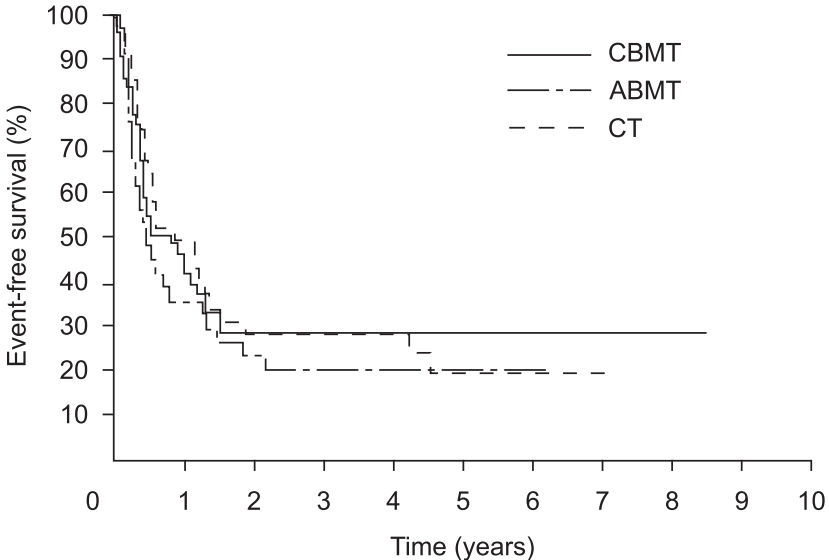

Figure 4 Estimated DFS from recovery of blood counts after remission induction compared by intention to treat analysis. Five-year DFS was similar with continued chemo (CT), conventional donorrelated HCT (CBMT) and alternative donor HCT (ABMT) using a matched unrelated donor, a haploidentical-related donor or 'purged' autologous hematopoietic stem cells (From Gaynon et al., Journal of Clinical Oncology 2006; 24: 3150. Reproduced with permission).

AML-directed chemo with chemo and allo-HCT in children and adolescents in the past decade illustrate that cure rates are equivalent or nearly so. The question remains as to whether selection bias with regard to socio-economic, insurance and ethnic factors favors the outcome of allo-HCT. In addition, lack of comparison of long-term morbidity and mortality of chemo versus allo-HCT hampers evaluation of their relative risk/benefit ratios.

The Children's Cancer Group led by Woods et al. ${ }^{26}$ randomized patients with AML in remission without matched family donors to continued chemotherapy or myeloablation and autologous HCT; 181 with available matched family donors were assigned to allo-HCT. Comparisons were made in accordance with 'intent to treat' analysis. Estimated 8-year overall survival for the children in remission was reported to be about $50 \%$ for the randomized patients but significantly higher for those assigned to allo-BMT. In a subsequent query it was pointed out that although Kaplan-Meier estimates showed better survival for allo-HCT; the plateaus of survival, which measure cure rate, were not significantly different. ${ }^{27}$ Others pointed out that the analysis was not a true intention to treat comparison. All donor-available patients were included in the analysis whether or not they received allo-HCT but not all patients without a donor were included, only those who were randomized between autologous HCT and chemo. ${ }^{28}$

A more recent report by Lange et al. $^{29}$ for the Children's Oncology Group describes the experience of 738 children and adolescents with AML in first remission treated in accordance with a more recent study protocol. The patients without available matched family donors were randomized to two alternate chemo regimens whereas 170 patients with donors were assigned to allo-HCT. Disease-free and overall survivals were within the same range for the two chemo regimens and for those observed in the chemotherapy alone arm of the earlier Woods et al. study. Comparison of allo-HCT with continued chemo by donor availability and intent to treat revealed no significant difference in actuarial 5-year overall survival but a moderate difference in disease-free survival (Figure 5). The report suggests that a subgroup of patients with AML and 'unfavorable cytogenetics' might benefit from allo-HCT. The authors say that


Figure 5 Estimated DFS and OAS of 170 children and adolescents with $\mathrm{AML}$ in first remission who had matched-related HCT donors and 463 who did not compared by intent-to-treat analysis. Five-year DFS was significantly superior for those with donors but 5 year OAS was similar (Figure 5a). However, for those with AML displaying historically 'favorable cytogenetics' the DFS as well as the OAS were similar (Figure 5b) (From Lange et al., Blood 2008; 111: 1044. Reproduced with permission).

allo-HCT is no longer being recommended for children and adolescents with AML that demonstrates 'favorable cytogenetics'.

The UK MRC AML 10 study of children with AML $<15$ years in first remission also compared outcome of extended chemo versus allo-HCT using donor availability and intent to treat. ${ }^{28}$ Of 85 children with a donor, 61 received allo-HCT. There was no difference in the 10-year survival between the donor and no donor patients. When results were combined with those of the subsequent AML 12 study in which 35 children received allo-HCT, there was no statistically different survival benefit for allo-HCT in any risk group, as classified by cytogenetics and response to initial chemo.

In BFM studies AML 87 and 93 reported by Creutzig et al., ${ }^{30}$ 418 children and adolescents with AML in first remission with 'high-risk' AML were assigned to allo-HCT, if they had matched family donors. When corrected for delay to transplant their 5 -year estimated disease-free and overall survival were similar for those who received continued chemo without transplant (Figure 6).

An EORTC report by Entze-Werle et al. ${ }^{31}$ compared allo-HCT and continued chemo in children in first remission of $\mathrm{AML}$ after intensification therapy. Using donor availability for comparison the 7-year-estimated disease-free and overall survival, were 


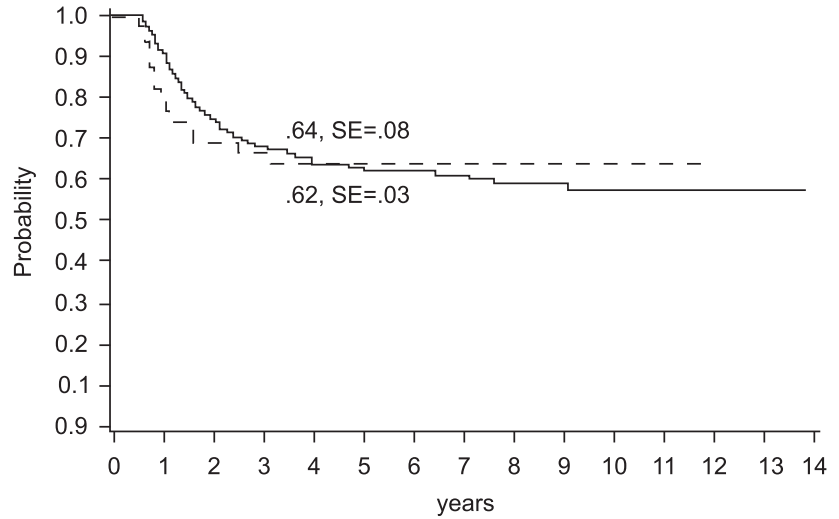

No MRD SCT ( $\mathrm{N}=317,121$ events)

- - - MRD SCT (N=39, 14 events)

Figure 6 Estimated EFS of children and adolescents $<18$ years in first remission of AML historically at 'high risk' of relapse. When corrected for time to transplant those assigned to matched-related donor HCT had similar EFS and OAS as those who received continued chemo without transplant (From Creutzig et al., Leukemia 2005; 19: 2030. Reproduced with permission).

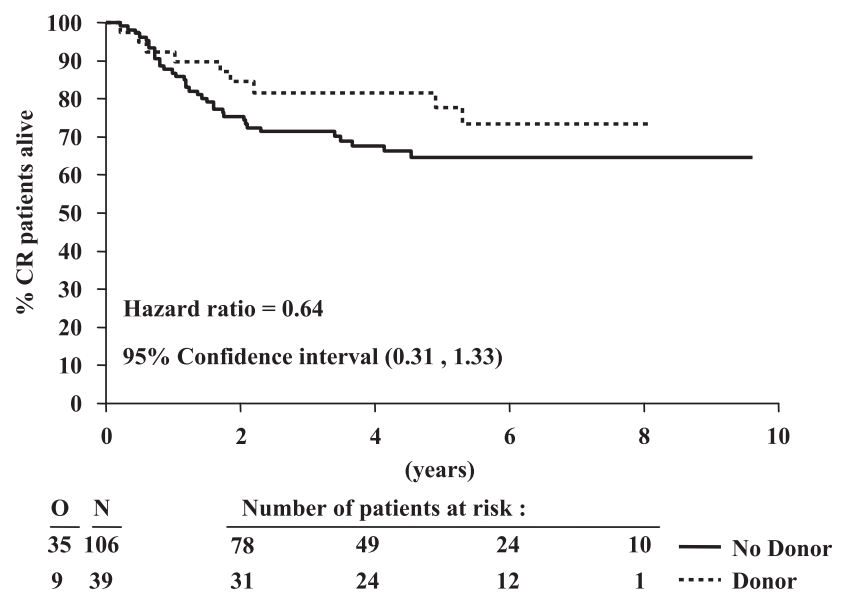

Figure 7 Estimated EFS of children and adolescents $<18$ years with AML in first remission, comparing Allo-HCT with continued chemo. Compared by donor availability and intention to treat the DFS and OAS from date of remission outcome was not significantly different (From Entz-Wehrle et al., Leukemia 2005; 19: 2072. Reproduced with permission. $\mathrm{N}=$ number of patients, $\mathrm{O}=$ observed number of deaths.).

similar for the 39 patients with a donor and the 106 who did not (Figure 7).

Results reported from the AEIOP group by Pession et al. ${ }^{32}$ in their studies of children less than 16 years old with AML in first remission differ from those of the EORTC. Correcting for median time from remission to transplant they found that estimated 5 -year disease-free survival was better for the patients who received allo-HCT than for those who received continued chemo alone. Overall survival is not reported so that eventual survival and presumed cure with chemo might be equivalent to that of allo-HCT as noted in ALL studies. ${ }^{12,14}$

With the exception of this report by the AIEOP group, information gathered in the past decade indicate that allo-HCT has no advantage in the practice of treating children with $A M L$, especially when allo-HCT's additional life-long risks of GVHD, growth failure, organ failure and carcinogenesis are considered.

\section{Discussion}

The story of allo-HCT exemplifies again the importance of conducting well designed and controlled, closely conducted and critically interpreted studies to determine the potential benefits and risks of new agents and procedures for patients before introducing them into clinical practice. This can take considerable self-discipline for bedside physicians because of our compassion and our stress when we are caring for sick children with fatal diseases. Like families we often want to 'grasp for straws', procedures that might help, without establishing or participating in a scientific assessment that will give an answer. When antifolates were shown to produce remission in some children with acute leukemia no controlled study was needed to conclude that they were beneficial because the rapidly progressive fatal course was well known. When the 4-phase total therapy program was shown in uncontrolled studies of small numbers of patients to produce cures, the curability of childhood leukemia was readily accepted. But randomized trials were then needed to test whether drug dosage needed to be maximal and more toxic and whether specific CNS therapy could be restricted to only those who showed leukemia cells in the cerebrospinal fluid at diagnosis or during chemo.

Allo-HCT for childhood leukemia was introduced in the 1970s because of reports of successful use in some children in remission with low expectations of survival and cure. ${ }^{33}$ As chemo improved in the 1980s with additional drugs, bolder combinations, better supportive care, more staff and more experience the use of allo-HCT in childhood leukemia also increased. There was clearly a need to test the relative benefits and risks of allo-HCT and the more modern chemo in well designed prospective comparative studies, randomizing patients just before the transplant procedure to avoid any of the possible selection factors mentioned earlier. However, considerable objection was raised that it would be unethical to withhold a possibly beneficial treatment from children with a high risk of death, an echo of the Arrowsmith dilemma. But actually the ethical violation is subjecting children to a very hazardous procedure with considerable risk of immediate, late and lifelong morbidity and mortality as well as child and family stress when the possible benefit has not been scientifically proven.

As described in this review and commentary, use of imperfect methods such as matched pairing, Mantel-Byar analysis and donor availability and intent-to-treat usually fail to demonstrate an advantage of using allo-BMT for children with acute leukemia when compared with contemporary chemo alone. As pointed out before, ${ }^{12,14}$ children who experience relapse after allo-HCT usually do not have further remissions whereas those who have relapses after chemo sometimes have subsequent lasting remissions. This results in overall survival of chemo-treated patients being greater than event-free and disease-free survival. Many published comparisons fail to provide information about overall survival. Further, the published comparisons tend to disregard the goal of treatment of ALL, long-term survival off treatment with minimal sequelae of the disease and its treatment. The plateaus of overall survival 4 years after cessation of therapy, the measure of cure of $A L L$, are not reported and compared. Cure, not palliation, has been the goal of ALL therapy for almost four decades.

Another major problem with most publications comparing allo-HCT versus continued chemo is the lack of information about late sequelae of treatment. This is essential to weighing the life-long hazards of a treatment against its benefits so that reasonable choices can be made by physicians, patients and parents when alternatives are available. In 2003 Pui et al. ${ }^{34}$ 


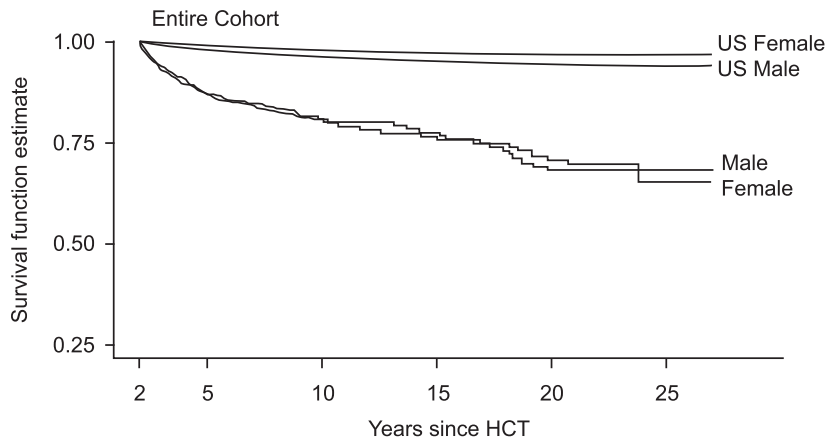

Figure 8 All cause mortality of 1479 two or more years survivors of all ages who received allo-HCT between 1974 and 1998, compared with age-adjusted expected survival rates for the US population. See reference for definition of survival function and its calculation (From Bhatia et al., Blood 2007; 110: 3784. Reproduced with permission).

described 856 survivors of ALL treated between 1962 and 1992. They concluded that those in 10-year complete remission who had not received preventive cranial irradiation can expect a normal long-term survival. This is supported by a 2000 report of 150 consecutive children with ALL treated between 1986 and $1994 .^{35}$ None received preventive cranial radiation nor simultaneous intravenous and intrathecal methotrexate, and only six received an anthracycline. Only three survivors had serious late adverse sequelae: personality disorder following asparaginaseinduced cortical vein thrombosis, chronic hepatitis $\mathrm{C}$ and osteonecrosis associated with pre-existing severe obesity.

Adverse sequelae of allo-HCT in children with leukemia are well known and described as noted above. ${ }^{5,6,7}$ Bhatia et al., ${ }^{36}$ more recently, described the late mortality and functional status of 1479 patients of all ages who received allo-HCT between 1974 and $1998 .^{37}$ As expected, mortality was excessive due to chronic graft versus host disease as well as recurrence of the original disease and second cancers; it remained twice that of the age-matched general population for more than 15 years after allo-HCT (Figure 8). In a subgroup of 547 patients compared with 319 sibling donors functional well being was reduced by all parameters, including marital status, employment and access to health and life insurance. For children, who have the potential of living many decades and all the challenges of growth and maturation to happy, healthy and productive adults, the use of allo-HCT for acute leukemia is especially difficult to justify. There is no proof that allo-HCT is more effective than chemo and abundant evidence that it is more harmful.

In 2005 the American Society for Blood and Bone Marrow Transplantation published a thorough detailed review of alloHCT in treatment of children with ALL. ${ }^{8}$ The report emphasizes the many current limitations to drawing conclusions about its value, including publication bias. As in this commentary, it points out the great need for high-quality randomized controlled trials with standardized methods, definitions of end points and reporting of study results. To this we add late as well as early results so that the long-term risks for the growing child can be weighed in with benefits for making treatment decisions.

Two issues pertinent to HCT generally, not just to its use in children, need to be mentioned. One is its application to futile care of patients with terminal disease such as drug refractory leukemia in relapse with widespread infection. No matter how optimistic the physician or how insistent the family, patients need to be protected from stressful interventions when the facts indicate its futility. Another issue is the diversion of scarce health care resources and personnel from basic preventive and primary care to the establishment and operation of expensive HCT units in economically distressed countries. On two occasions I visited expensive HCT units in developing nations where at the same location children were dying of highly curable B-precursor ALL, because they were denied access to relatively low-cost drugs like prednisone, methotrexate and mercaptopurine. A somewhat analogous situation can be seen in a relatively prosperous developed nation where highly costeffective basic child and maternal health is often neglected, whereas less cost-effective specialized care flourishes.

\section{Conflict of interest}

The author declares no conflict of interest.

\section{Acknowledgements}

Many thanks to Ching-Hon Pui, MD, Paul Gaynon, MD and Joseph Simone, MD for their reviews, suggestions and assistance in preparation of this paper. The views expressed are those of the author.

${ }^{1}$ Department of Biological Sciences, College of Science and Mathematics, California Polytechnic State University, San Luis Obispo, CA, USA and

${ }^{2}$ St Jude Children's Research Hospital, Memphis, TN, USA E-mail: donpinkel@yahoo.com

\section{References}

1 Pinkel D. Allogeneic bone marrow transplantation in children with acute leukemia: a practice whose time has gone. Leukemia 1989; 3: 242-244.

2 Chessells J. A reply to D Pinkel, allogeneic bone marrow transplantation in childhood leukemia: another form of intensive treatment. Leukemia 1989; 3: 543-544.

3 Kersey JH. Reply to Donald Pinkel. Leukemia 1989; 3: 394-395.

4 Pinkel D. Bone marrow transplantation in children with acute leukemia: a 1990 view. Haematol.Bluttransf 1992; 35: 3-10.

5 Pinkel D. Bone marrow transplantation in children. J Peds 1993; 122: 331-341.

6 Deeg $\mathrm{H}$. Bone marrow transplantation: A review of delayed complications. Br J Haematol 1984; 57: 185-208.

7 Sanders J, Pritchard S, Mahoney P, Amos D, Buckner D, Witherspoon $\mathrm{R}$ et al. Growth and development following marrow transplantation for leukemia. Blood 1986; 5: 1129-1135.

8 Hahn T, Wall D, Camitta B, Davies S, Dillon H, Gaynon P et al. The role of cytotoxic therapy with hematopoietic stem cell transplantation in the therapy of acute lymphoblastic leukemia in children: An evidenced-based review. Biol Blood Bone Marrow Transplant 2005; 11: 823-861.

9 Pui C-H, Gaynon P, Boyett J, Chessells J, Baruchel A, Kamps W et al. Outcome of treatment in childhood acute lymphoblastic leukemia with rearrangements of the $11 \mathrm{q} 23$ chromosomal region. The Lancet 2002; 359: 1909-1915.

10 Chessells J, Harrison C, Watson S, Vora A, Richards S. Treatment of infants with lymphoblastic leukemia: results of the UK infant protocols 1987-1999. Br J Haematol 2002; 117: 306-314.

11 Pieters R, Schrappe M, De Lorenzo P, Hann I, De Rossi G, Felice $M$ et al. A treatment protocol for infants younger than 1 year with acute lymphoblastic leukemia (Interfant-99): an observational study and a muticentre randomised trial. The Lancet 2007; 370: 240-250.

12 Balduzzi A, Valsecchi M, Uderzo C, De Lorenzo P, Klingebiel T, Peters $C$ et al. Chemotherapy versus allogeneic transplantation in first complete remission for very high-risk childhood acute lymphoblastic leukemia in first complete remission: comparison by genetic randomization in an international prospective study. The Lancet 2005; 366: 635-642. 
13 Arico M, Valsecchi M, Camitta B, Schrappe M, Chessells J, Baruchel A et al. Outcome of treatment in children with Philadelphia chromosome-positive acute lymphoblastic leukemia. N Engl J Med 2000; 342: 998-1006.

14 Arico M, Schrappe M, Hunger S, Carroll W, Conter V, Di Lorenzo $\mathrm{P}$ et al. Clinical outcome of 640 children with newly diagnosed Philadelphia chromosome-positive acute lymphoblastic leukemia treated between 1995 and 2005. Blood (ASH annual meeting abstracts) 2008; 112. abstract 568.

15 Schultz K, Bowman WP, Slayton W, Aledo A, Devidas M, Sather H et al. Improved early event free survival with imatinib in Philadelphia chromosome-positive acute lymphoblastic leukemia: a Children's Oncology Group Study. J Clin Oncol (in press).

16 Nachman J, Heerema N, Sather H, Camitta B, Forestier E, Harrison $\mathrm{C}$ et al. Outcome of treatment in children with hypodiploid acute lymphoblastic leukemia. Blood 2007; 110: 1112-1115.

17 Nguyen K, Devidas M, Cheng S-C, La M, Raetz E, Carroll W et al. Factors influencing survival after relapse from acute lymphoblastic leukemia: A Children's Oncology Group Study. Leukemia 2008; 22: 2142-2150.

18 Lawson S, Harrison G, Richards S, Oakhill A, Stevens R, Eden O et al. The UK experience in treating relapsed childhood acute lymphoblastic leukemia: a report on the Medical Research Council UKALLR1 study. Br J Haematol 2000; 108: 31-543.

19 Eapen M, Raetz E, Zhang M-J, Muehlenbein C, Devidas M, Abshire $\mathrm{T}$ et al. Outcomes after HLA-matched sibling transplantation or chemotherapy in children with B-precursor acute lymphoblastic leukemia in a second remission: a collaborative study of the Children's Oncology Group and the Center for International Blood and Marrow Transplant Research. Blood 2006; 107: 4961-4967.

20 Einsiedel H, Von Stackelberg A, Hartmann R, Fengler R, Schrappe $M$, Janka-Schaub $G$ et al. Long term outcome in children with relapsed ALL by risk-stratified salvage therapy: result of trial acute lymphoblastic leukemia-relapse study of the Berlin-FrankfurtMunster Group 87. J Clin Oncol 2005; 23: 7942-7950.

21 Schroeder H, Gustaffson G, Saarinen-Pikhala U, Glomstein A, Jonmundsson $\mathrm{G}$, Nysom $\mathrm{K}$ et al. Allogeneic bone marrow transplantation in second remission of childhood acute lymphoblastic leukemia: a population-based case-control study from the Nordic countries. Bone Marrow Transplant 1999; 23: 555-560.

22 Saarinen-Pikhala U, Heilmann C, Winiarski J, Glomstein A, Abrahamsson J, Arvidson J et al. Pathways through relapses and deaths of children with acute lymphoblastic leukemia: role of allogeneic stem cell transplantation in Nordic data. J Clin Oncol 2006; 24: 5750-5762.

23 Roy A, Cargill A, Love S, Moorman A, Stoneham S, Lim A et al. Outcome after first relapse in childhood acute lymphoblastic leukemia-lessons from the United Kingdom R2 Trial. BrJ Haematol 2005; 130: 67-75.

24 Malempati S, Gaynon P, Sather H, La M, Stork L. Outcome after relapse among children with standard risk acute lymphoblastic leukemia: Children's Oncology Group Study CCG-1952. J Clin Oncol 2007; 25: 5800-5807.
25 Gaynon P, Harris R, Altman A, Bostrom B, Breneman J, Hawks R et al. Bone marrow transplantation versus prolonged intensive chemotherapy for children with acute lymphoblastic leukemia and an initial bone marrow relapse within 12 months of the completion of primary therapy: Children's Oncology Group Study CCG-1941. J Clin Oncol 2006; 24: 3150-3156.

26 Woods W, Neudorf S, Gold S, Sanders J, Buckley J, Barnard D et al. A comparison of allogeneic bone marrow transplantation, autologous bone marrow transplantation, and aggressive chemotherapy in children with acute myeloid leukemia in remission: a report from the Children's Cancer Group. Blood 2001; 97: 56-62.

27 Pinkel D. Letter to the editor: treatment of children with acute myeloid leukemia. Blood 2001; 97: 3673.

28 Gibson B, Wheatley K, Hann I, Stevens R, Webb D, Hills R et al. Treatment strategy and long term results in paediatric patients treated in consecutive UK AML trials. Leukemia 2005; 19: $2130-2138$.

29 Lange B, Smith F, Feusner J, Barnard D, Dinndorf P, Feig S et al. Outcomes in CCG-2961, a Children's Oncology Group Phase 3 trial for untreated pediatric acute myeloid leukemia: A Report from the Children's Oncology Group. Blood 2008; 111: 1044-1053.

30 Creutzig U, Zimmerman M, Ritter J, Reinhardt D, Hermann J, Henze G et al. Treatment strategies and long term results in paediatric patients treated in four consecutive AML-BFM trials. Leukemia 2005; 19: 2030-2042.

31 Entz-Werle N, Suciu S, Van der Werff ten Bosch J, Vilmer E, Bertrand Y, Benoit $Y$ et al. Results of 58872 and 58921 trials in acute myeloblastic leukemia and relative value of chemotherapy vs allogeneic bone marrow transplantation in first complete remission: The EORTC Children's Leukemia Group Report. Leukemia 2005; 19: 2072-2081.

32 Pession A, Rondelli R, Basso G, Rizzari C, Testi AM, Fagioli F et al. Treatment and long-term results in children with acute myeloid leukemia treated according to the AIEOP AML protocols. Leukemia 2005; 19: 2043-2053.

33 Thomas ED, Buckner C, Rudolph R, Fefer A, Storb R, Neiman P et al. Allogeneic marrow grafting for hematologic malignancy using HL-A matched donor recipient sibling pairs. Blood 1971; 38 : 267-287.

34 Pui $\mathrm{CH}$, Cheng C, Leung W, Rai S, Rivera G, Sandlund J et al. Extended follow-up of long-term survivors of childhood acute lymphoblastic leukemia. N Engl J Med 2003; 349: 640-649.

35 Manera R, Ramirez I, Mullins J, Pinkel D. Pilot studies of speciesspecific chemotherapy of childhood acute lymphoblastic leukemia using genotype and immunophenotype. Leukemia 2000; 14: 1354-1361.

36 Bhatia S, Francisco L, Carter A, Sun C-L, Baker KS, Gurney JG et al. Late mortality after allogeneic hematopoietic cell transplantation and functional status of long term survivors: Report from the Bone Marrow Transplant Survivor Study. Blood 2007; 110: 3784-3792.

37 Tichelli A. Comment: hematopoietic cell transplantation: a lifelong commitment. Blood 2007; 110: 3493-3494. 